\title{
Acquisition of Pseudomonas cepacia at summer camps for patients with cystic fibrosis
}

\author{
David A. Pegues, MD, Loretta A. Carson, MS, Ofelia C. Tablan, MD, \\ Stacey C. FitzSimmons, PhD, Susan B. Roman, MMSc, J. Michael Miller, PhD, \\ William R. Jarvis, MD, and the Summer Camp Study Group ${ }^{a}$
}

To assess the risk of acquisition of Pseudomonas cepacia by person-to-person transmission at cystic fibrosis summer camps, we conducted in 1990 a study at three camps attended by patients with cystic fibrosis who had $P$. cepacia infection and patients without $P$. cepacia infection but who were considered susceptible to infection. We obtained sputum or throat cultures from campers on their arrival at, weekly during, at the end of, and 14 to 30 days after camp. We compared the incidence of sputum conversion of patients at camp with that of patients outside camp by culturing specimens from noncamper control subjects with cystic fibrosis who were known not to be infected $\leq 2$ weeks before and 4 to 6 weeks after camp. We also determined the risk factors for $P$. cepacia acquisition by determining the relative risk of acquisition between campers who were exposed versus campers who were not exposed to campers known to be infected or to potential environmental sources of $P$. cepacia at camp. The ribotype of $P$. cepacia isolates from campers with sputum conversion was compared with that of isolates from other campers and from an environmental source. The cumulative incidence of sputum conversion during the study period was $6.1 \%(11 / 181)$ among campers compared with no incidence $(0 / 92)$ among noncampers $(p=0.02$, Fisher Exact Test). The incidence of sputum conversion at camp varied according to the prevalence of campers with known infection ( $p<0.001$, chi-square test for trend). The rate of sputum conversion was higher in the camp with longer duration (relative risk $=12.0 ; 95 \%$ confidence inter$\mathrm{val}=2.7$ to 53.5). Ribotyping showed that $P$. cepacia isolates from all $11 \mathrm{camp}$ ers with sputum conversion were identical or simllar ( 1 to 2 band difference) to isolates of other $P$. cepacia-infected campers including co-converters. These results suggest that $P$. cepacia can be acquired by patients with cystic fibrosis who are attending summer camp for such patients, possibly through person-toperson transmission, and that the risk increases with the prevalence of $P$. cepacia-infected campers and the duration of camp. (J PeDIATR 1994;124:694-702)

Supported by a grant from the Cystic Fibrosis Foundation.

Presented in part at the 31 st Interscience Conference on Antimicrobial Agents and Chemotherapy, Chicago, Ill., Sept. 29 to Oct. 2, 1991; and the 1991 North American Cystic Fibrosis Conference, Dallas, Texas, Oct. 2-5, 1991.

Submitted for publication Sept. 10, 1993; accepted Dec. 13, 1993.
Reprint requests: Ofelia C. Tablan, MD, Hospital Infections Program, Centers for Disease Control and Prevention, MS A 07, 1600 Clifton Rd., NE, Atlanta, GA 30333.

aParticipants in the Summer Camp Study Group are listed immediately after the text.

Copyright ${ }^{\odot} 1994$ by Mosby-Year Book, Inc. $0022-3476 / 94 / \$ 3.00+0 \quad \mathbf{9} / \mathbf{2 0} / \mathbf{5 3 5 4 8}$ 
Pseudomonas cepacia, a multi-drug-resistant gram-negative bacillus, has been associated with chronic colonization of the respiratory tract of patients with cystic fibrosis, and in some patients has been associated with rapid decline in pulmonary function, increased hospitalization, and earlier death. ${ }^{1-6}$ In 1990 P. cepacia was isolated from $3.2 \%$ of all patients' cultures from a national registry of patients with $\mathrm{CF},{ }^{7}$ and the incidence of new acquisition of $P$. cepacia has been estimated to be $1.5 \%$ per year (S. FitzSimmons: personal communication, 1992). Studies suggest that personto-person transmission of $P$. cepacia is one possible mode of acquisition in and outside the hospital setting; however, the risk of acquiring $P$. cepacia at $\mathrm{CF}$ summer camps by a patient with CF has not been carefully studied. ${ }^{2,8-11}$ Because of concern that patients attending CF summer camps may be at increased risk of acquiring $P$. cepacia, we investigated three summer camps from June to August 1990 to determine the incidence of, risk factors for, and possible modes of acquisition of $P$. cepacia by patients with CF at camp.

\section{BACKGROUND}

Three camps attended by a total of 270 patients with CF were studied. All three camps had campers and counselors known to have $P$. cepacia infection, but the prevalence of $P$. cepacia-infected patients differed.

Camp A-Ohio. From June 9 to 16, 1990, an annual 7-day camp was held for patients with $\mathrm{CF}$ from three $\mathrm{CF}$ centers in Ohio and one in Kentucky. Patients aged 6 to 14 years were eligible as campers; 15 - to 17 -year-old patients were admitted as counselors-in-training, and patients $\geq 18$ years old were "staff members." Campers were housed in groups of five to nine individuals, according to age and sex, in 10 separate cabins. Each camper had one staff member who supervised him or her 24 hours a day, administered respiratory therapy, and performed chest physiotherapy. A total of 87 patients with CF, including three $P$. cepacia-infected patients, attended Camp A.

Camp B-Utah. From July 21 to 28, 1990, a 7-day camp was conducted for patients from one CF center in Utah. Patients 6 to 45 years of age were eligible. Most campers were housed according to age and sex, in groups of two to six, in 15 separate cabins or tents. Each group was supervised by one or more staff members. Some campers stayed in private tents or camping vehicles with family members. A total of 67 patients with $\mathrm{CF}$, including 10 who had $P$. cepacia infection, attended Camp B.

Camp C-Ontario. From August 4 to 31, 1990, a 28-day camp was sponsored for patients from four CF Centers in Ontario. Patients aged 5 to 14 years were eligible as campers, patients aged 14 to 17 years were admitted as counselors/staff-in-training, and patients aged $\geq 18$ years were employed as counselors/staff members. Campers were grouped according to age and sex, 5 to 10 members in each group, housed by group in 13 cabins, and supervised by two or three staff members per group. A total of 114 patients, including 37 known to have $P$. cepacia infection, attended Camp C.

At each of the three study camps, campers participated in many outdoor and indoor activities, including swimming, other water sports, and dancing. Cabin mates shared many activities and were not restricted from mingling with campers from other cabins. Individualized medical care, including both intravenous and oral administration of medications, respiratory therapy, and chest physiotherapy, was provided by volunteer medical, paramedical, and lay staff members according to instructions from patients' attending physicians. Hand washing before and after chest physiotherapy and after handling potentially contaminated articles was not routinely performed, and patients were not assigned to a cohort according to their $P$. cepacia-colonization status. Aerosol therapy and chest physiotherapy were administered one to four times each day in each camper's cabin or in a common area. Each camper used his or her own medication nebulizer daily.

\begin{tabular}{|ll|}
\hline CF & Cystic fibrosis \\
CI & Confidence interval \\
EDTA & Ethylenediaminetetraacetic acid \\
RR & Relative risk \\
\hline
\end{tabular}

\section{METHODS}

\section{Identifying the study patients}

Campers. Informed consent was obtained from each patient with $C F$ who was $\geq 18$ years of age or from each minor patient's guardian. To determine colonization status, we obtained a sputum or throat culture specimen from each consenting patient on arrival at camp, and the results of the previous sputum cultures were obtained from the patient's CF center physician. A camper was considered uninfected if his or her culture was negative for $P$. cepacia on arrival at camp, if at least one sputum/throat culture specimen per year had been obtained during the 2 years preceding camp, and if no previous culture had been positive for $P$. cepacia. Five campers (two from Camp A and three from Camp C) who were uninfected on arrival and during camp, but who had at least one culture positive for $P$. cepacia during the 2 years preceding camp, were excluded from the analysis. A camper was considered infected if he or she had positive culture results for $P$. cepacia on arrival at camp.

Noncampers. Noncamper control subjects were patients with negative culture results for $P$. cepacia who attended the same CF centers as campers, had received outpatient or in- 
patient care at their respective $\mathrm{CF}$ centers during the 2-week period before camp, and who had never previously had a culture positive for $P$. cepacia, including any of at least three sputum or throat cultures (one per year in the preceding 2 years, and one during the 2-week period before the respective $\mathrm{CF}$ center's summer camp started).

Incidence of $\boldsymbol{P}$. cepacia acquisition, campers versus noncampers. Acquisition of $\boldsymbol{P}$. cepacia by campers was determined by obtaining sputum or throat culture specimens from uninfected campers at the end of the 7-day camps (Camp A, Camp B), or weekly at the 28-day camp (Camp C), and on follow-up at 14 to 30 days after the end of camp. For noncampers, follow-up sputum or throat culture specimens also were obtained 14 to 30 days after the last day of the respective camps. We compared the cumulative incidence of sputum conversion in campers at the end of camp and at the end of the follow-up period with the incidence of sputum conversion in noncampers.

Potential exposures at camp. To evaluate potential risk factors for sputum conversion at camp, we asked all participating campers, their respective counselors, or both to complete questionnaire forms daily at Camps $\mathrm{A}$ and $\mathrm{B}$, or weekly at Camp C. The questionnaire assessed each camper's close contact with other campers and staff members, frequency of respiratory therapy and chest physiotherapy, frequency of hand washing, and potential exposure to other potential environmental sources of $P$. cepacia. In addition, for each uninfected camper and noncamper, the respective CF center director completed a follow-up questionnaire regarding the patient's age, gender, severity of $\mathrm{CF}$, respiratory culture results from 1988 and 1989, and potential exposures to patients with culture findings positive for $P$. cepacia, to environmental sources of $P$. cepacia, and to antipseudomonal antimicrobial agents received during the follow-up period.

Statistical methods. The point estimate of the relative risk of sputum conversion in campers compared with noncampers and its $95 \%$ confidence interval were calculated. The chi-square test for trends was used to determine the linear association between the incidence of sputum conversion and the prevalence of previously known $P$. cepacia-infected campers. Proportions were compared with the Fisher Exact Test or the chi-square test where appropriate. In the analysis of camp-associated risk factors for sputum conversion, the point RR and its 95\% CI were calculated for categoric variables. The Mann-Whitney rank sum test was used to compare the difference in mean age between the campers and noncampers.

Collection, transport, and processing of specimens. At camp, investigators obtained sputum or throat culture specimens from all study patients after the patients' morning chest physiotherapy. Sputum or throat culture speci- mens were obtained from noncamper control subjects during their outpatient visit or hospitalization within the 2-week period before the start of the respective CF center's camp.

A throat swab consisted of a complete swab of the posterior, anterior, and lateral portions of the patient's pharyngeal wall with a sterile throat swab, performed immediately after the patient attempted to cough. Throat swabs were placed into transport medium. Sputum specimens were collected in sterile screw-capped containers. All specimens were packed securely and shipped at ambient temperature overnight to the Centers for Disease Control and Prevention (CDC). Excellent recovery of $P$. cepacia organisms has been demonstrated in sputum culture specimens maintained at $25^{\circ} \mathrm{C}$ to $30^{\circ} \mathrm{C}$ for up to 14 days (CDC: unpublished data).

Follow-up culture specimens were obtained from campers and noncampers during their outpatient visit or hospitalization between 14 and 30 days after camp by CF center health care workers. Patients who did not return to their respective $\mathrm{CF}$ centers during the 14 to 30 days after camp were each given a sputum collection cup and a throat swab and were directed either to collect a sputum specimen at home or to have a throat culture specimen obtained by a local health care worker. Patients airmailed their culture specimens to the CDC.

Immediately on arrival at the $\mathrm{CDC}$, sputum specimens were homogenized in a vortex mixer for 2 minutes. A sterile cotton swab was dipped into the homogenized sputum and swirled 12 times, and then the sputum was inoculated onto sheep blood-agar and $P$. cepacia-agar plates (BBL Microbiology Systems, Cockeysville, Md.). ${ }^{12}$ Throat swabs were directly inoculated onto blood-agar and $P$. cepaciaagar plates. Plates were incubated at $35^{\circ} \mathrm{C}$ for up to 96 hours and examined daily. Phenotypically different strains were harvested separately and identified individually. Microorganisms were identified by means of conventional and automated biochemical tests (MicroScan Walk/Away, Baxter Diagnostics, Inc., Microscan Division, West Sacramento, Calif.*). ${ }^{13}$

Water from pools, lakes, drinking fountains, sinks, and showers at each camp was collected in $500 \mathrm{ml}$ samples into sterile containers and shipped at ambient temperature overnight to the CDC. At the CDC, viable cell counts were determined by the membrane filtration technique. ${ }^{14}$ Aliquots of $1 \mathrm{ml}$ and $50 \mathrm{ml}$ of 10 -fold serial dilutions of each water specimen were passed through $0.45 \mu \mathrm{m}$ membrane filters, and the filters were inoculated onto MacConkey-,

*Use of trade names and commercial sources is for identification only and does not imply endorsement by the Public Health Service or the U.S. Department of Health and Human Services. 
Table. Assessment of potential risk factors for acquisition of $P$. cepacia among CF campers in Ohio, Utah, and Ontario in 1990

\begin{tabular}{|c|c|c|c|c|}
\hline \multirow[b]{2}{*}{ Exposure } & \multicolumn{2}{|c|}{ P. cepacia acquisition among campers } & \multirow[b]{2}{*}{$\mathbf{R R}$} & \multirow[b]{2}{*}{$(95 \% \mathrm{Cl})$} \\
\hline & $\begin{array}{c}\text { Exposed } \\
\text { proportion (\%) }\end{array}$ & $\begin{array}{c}\text { Unexposed } \\
\text { proportion (\%) }\end{array}$ & & \\
\hline $\begin{array}{l}\text { Shared item with } P \text {. cepacia-infected } \\
\text { camper }\end{array}$ & $3 / 9(33)$ & $6 / 161(4)$ & 8.9 & $2.7-30.1$ \\
\hline Danced with $P$. cepacia-infected camper & $3 / 18(17)$ & $6 / 152(4)$ & 4.2 & $1.2-15.4$ \\
\hline $\begin{array}{l}\text { Slept in cabin with } P \text {. cepacia-infected } \\
\text { camper }\end{array}$ & $7 / 61(11)$ & $4 / 130(3)$ & 3.7 & $1.1-12.3$ \\
\hline Kissed $P$. cepacia-infected camper & $1 / 21(5)$ & $8 / 149(5)$ & 0.9 & $0.1-6.7$ \\
\hline Hugged $P$. cepacia-infected camper & $4 / 45(9)$ & $5 / 125(4)$ & 2.2 & $0.6-7.9$ \\
\hline Ate any fresh fruit or vegetables & $7 / 164(4)$ & $2 / 6(33)$ & 0.1 & $0.03-0.5$ \\
\hline Washed hands $\leq 3$ times a day & $3 / 68(4)$ & $6 / 102(6)$ & 0.8 & $0.2-2.9$ \\
\hline
\end{tabular}

blood-, and $P$. cepacia-agar plates. Culture plates were incubated at $35^{\circ} \mathrm{C}$ for up to 96 hours and examined daily for growth of organisms.

Ribotyping of $\boldsymbol{P}$. cepacia. By means of ribotyping, a technique for detecting DNA fragments carrying ribosomal ribonucleic acid genes, we compared phenotypically distinct isolates from patients who became infected with isolates from all known $P$. cepacia-infected camp attendees participating in the study. Preliminary experiments were done with DNA extracts from four strains used to serotype $P$. cepacia by use of restriction digests of $\mathrm{BamHI}, \mathrm{Bgl}$ II, Cla I, EcoRI, HindIII, Not I, Pst I, Pvu I, Sfi I, and Sma I to select the enzyme or enzymes that provide the best discrimination of hybridization banding patterns. ${ }^{14 a}$ Ribotyping analysis of the $\boldsymbol{P}$. cepacia isolates from study patients was subsequently performed by use of EcoRI and Cla I restriction digests. The resulting fragments were separated by gel electrophoresis in $1.0 \%$ agarose in Tris-acetate-EDTA buffer, and transferred onto a nylon membrane (MagnaGraph Nylon, Micron Separations, Inc., Westborough, Mass.). Membranes were probed with digoxigenin-labeled complementary DNA derived from reverse transcription of 16S/23S ribosomal RNA; hybridization bands were detected directly by an immunologic method (Genius System, Boehringer-Mannheim, Indianapolis, Ind.). Because Cla I digests, which produced 9 to 12 bands, provided greater discriminatory capacity than $E c o$ RI digests, which typically yielded 5 to 10 bands, they were used for the final determination of relatedness of isolates from patients with $P$. cepacia sputum conversion and other camp attendees.

\section{RESULTS}

Of the 270 campers, $237(88 \%)$ agreed to participate in the study. (The 33 nonparticipants were similar to the participants in age and sex. Four [12\%] had $P$. cepacia infection before camp; all four attended Camp C.) Of the 237 participants, $191(81 \%)$ had cultures negative for $P$. cepacia on arrival at camp and had never previously had cultures positive for $P$. cepacia. The median age of these study patients was 12 years (range, 5 to 45 years); 98 (51\%) were male subjects. Of the participating campers, $46(19 \%)$ had culture findings positive for $P$. cepacia. The prevalence of $P$. cepacia-infected campers at the beginning of camp varied among the three camps: $3(3.4 \%)$ of 87 at Camp A, 10 (15.9\%) of 63 at Camp B, and $33(37.9 \%)$ of 87 at Camp C.

Incidence of acquisition of $\boldsymbol{P}$. cepacia. Of 191 campers with initial culture findings negative for $P$. cepacia, 10 ( $5.2 \% ; 95 \% \mathrm{CI}=2.7 \%$ to $9.7 \%$ ) had one or more positive culture results during camp (range: 1 to 4 positive culture results per converter). Sputum or throat specimens cultured 14 to 30 days after camp for $181(95 \%)$ of 191 campers with initially negative results showed that one additional camper had sputum conversion. This camper had five weekly cultures negative for $P$. cepacia while at Camp $\mathrm{C}$ but a positive culture result 2 weeks after camp. Thus the cumulative incidence of sputum conversion in campers with results negative for $P$. cepacia who completed the follow-up was 11 (6.1\%) of $181(95 \% \mathrm{CI}=3.2 \%$ to $10.9 \%)$.

The risk of sputum conversion in campers with negative culture results increased with an increase in the prevalence of $P$. cepacia-infected persons at camp: none of the 84 at Camp A (prevalence of $P$. cepacia-infected campers: $3.4 \%$ ); 2 (4.3\%) of 47 at Camp B (prevalence: $15.9 \%$ ); and $9(18.0 \%)$ of 50 at Camp C (prevalence: $37.9 \%)(p<0.001$, chi-square test for trend). In addition, the risk of sputum conversion was significantly higher in the camp with the longer duration: 9 (18\%) of 50 persons at Camp C (duration: 4 weeks) versus $2(1.5 \%)$ of 131 at Camp A and Camp $\mathrm{B}$ (duration: 1 week each) $(\mathrm{RR}=12.0 ; 95 \% \mathrm{CI}=2.7 \%$ to $53.5 \%$ ).

In addition to the camper whose sputum conversion was 


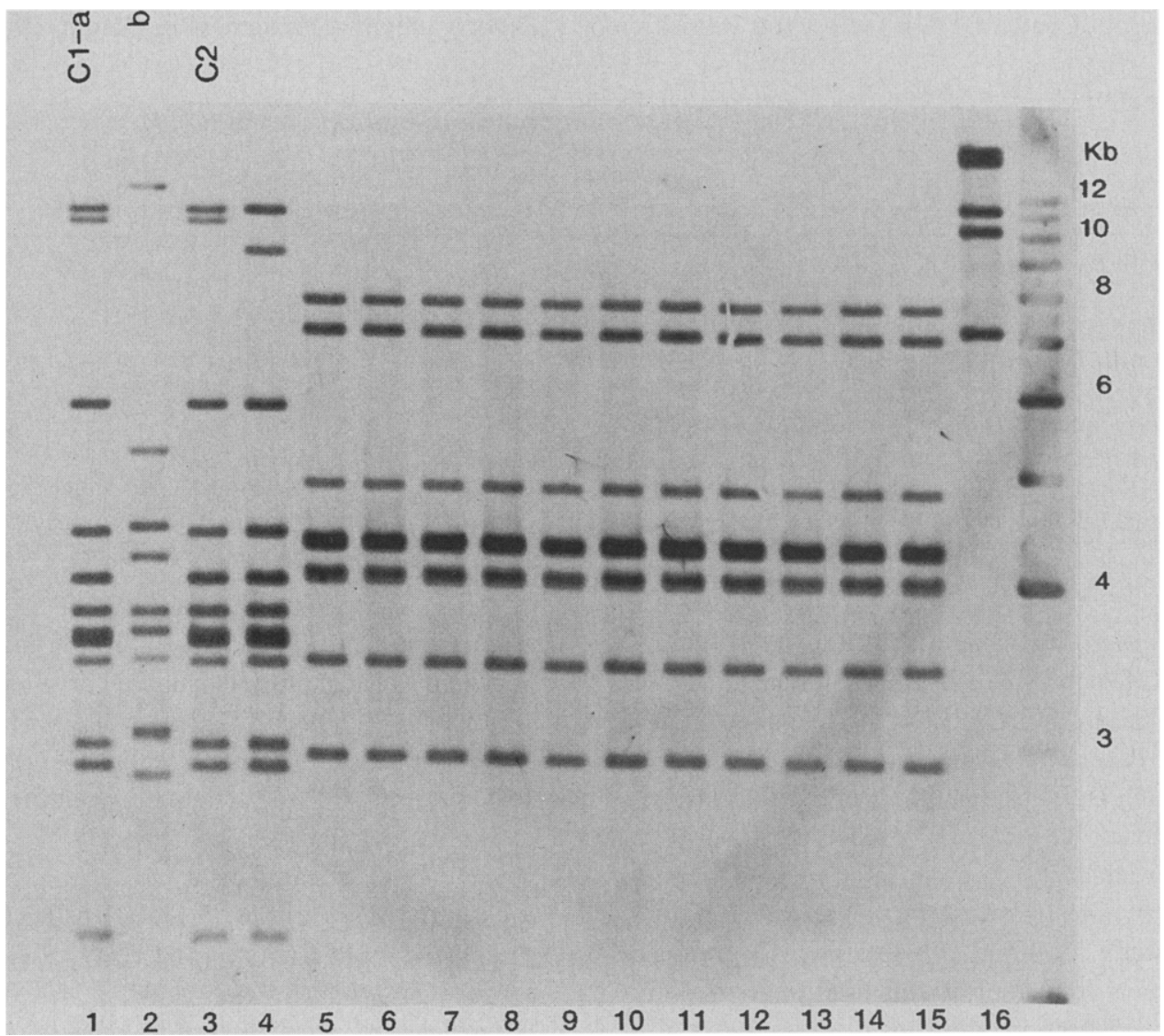

Fig. 1. Ribotyping of $C l a$ I-digested DNA from $P$. cepacia isolates, Camp B, Utah. $P$. cepacia isolates from the first subject with sputum conversion (lanes 1 and 2 , marked $C 1-a$ and $C I-b$, respectively), the second with conversion $(C 2$, lane 3), $12 P$. cepacia-infected campers (lanes 4 to 15), and water from a picnic jug (lane 16). Size markers in kilobase pairs = migration of HindIII-digested lambda phage DNA (lane 17). One of two P. cepacia isolates from the first subject with sputum conversion ( $\mathrm{Cl}$-a, lane 1$)$ had the same banding pattern as the isolate from the second subject with conversion (C2, lane 3) and was very similar to (i.e., had one band different from, and 11 bands identical with) the ribotype of an isolate from a known $P$. cepacia-infected camper (lane 4) with whom they reported close contact. The first converter's second $P$. cepacia isolate had a unique ribotype $(C 1-b$, lane 2$)$ that was different from all other ribotypes found at that camp.

first detected 2 weeks after camp, only 3 of the 10 subjects with sputum conversion at camp had a culture positive for $P$. cepacia on follow-up. Thus if a point estimate of the incidence of sputum conversion in campers had been done only at the end of the follow-up period (as was done for noncampers), it would have been $4(2.2 \%)$ of $181(95 \%$ $\mathrm{CI}=0.7 \%$ to $5.9 \%$ ).

During 12 additional months of observation, $P$. cepacia was recovered intermittently from sequential respiratory cultures from $4(36 \%)$ of the 11 subjects with sputum conversion (range, 2 to 4 positive culture results per converter).

Association between attendance at camp and acquisition of $\boldsymbol{P}$. cepacia. None of 92 noncamper control subjects with follow-up cultures had a sputum conversion (upper limit of $95 \% \mathrm{CI}=5.0 \%$ ); thus the cumulative incidence of sputum conversion by the end of the 4-week follow-up period was significantly higher in campers than in noncampers (11 [6.1\%] of 181 vs none of $92 ; p=0.02$, Fisher Exact Test). However, if the analysis had been restricted to the follow-up cultures alone, the incidence of $P$. cepacia acquisition among campers and noncampers would not have been significantly different (4 [2.2\%] of 181 vs none of $92 ; p=0.30$, Fisher Exact Test).

In comparing campers with negative culture results and noncamper control subjects to determine whether the higher incidence of sputum conversion in campers may have been partly the result of differences in patient characteristics, we found that campers with negative culture results were significantly younger (mean age: 11.8 years vs 13.5 years; $p=0.02$ ) and had milder underlying CF (mild CF: $103[61 \%]$ of 170 vs 36 [39\%] of $92 ; p<0.001$, chi-square test). However, the two patient groups had similar sex dis- 


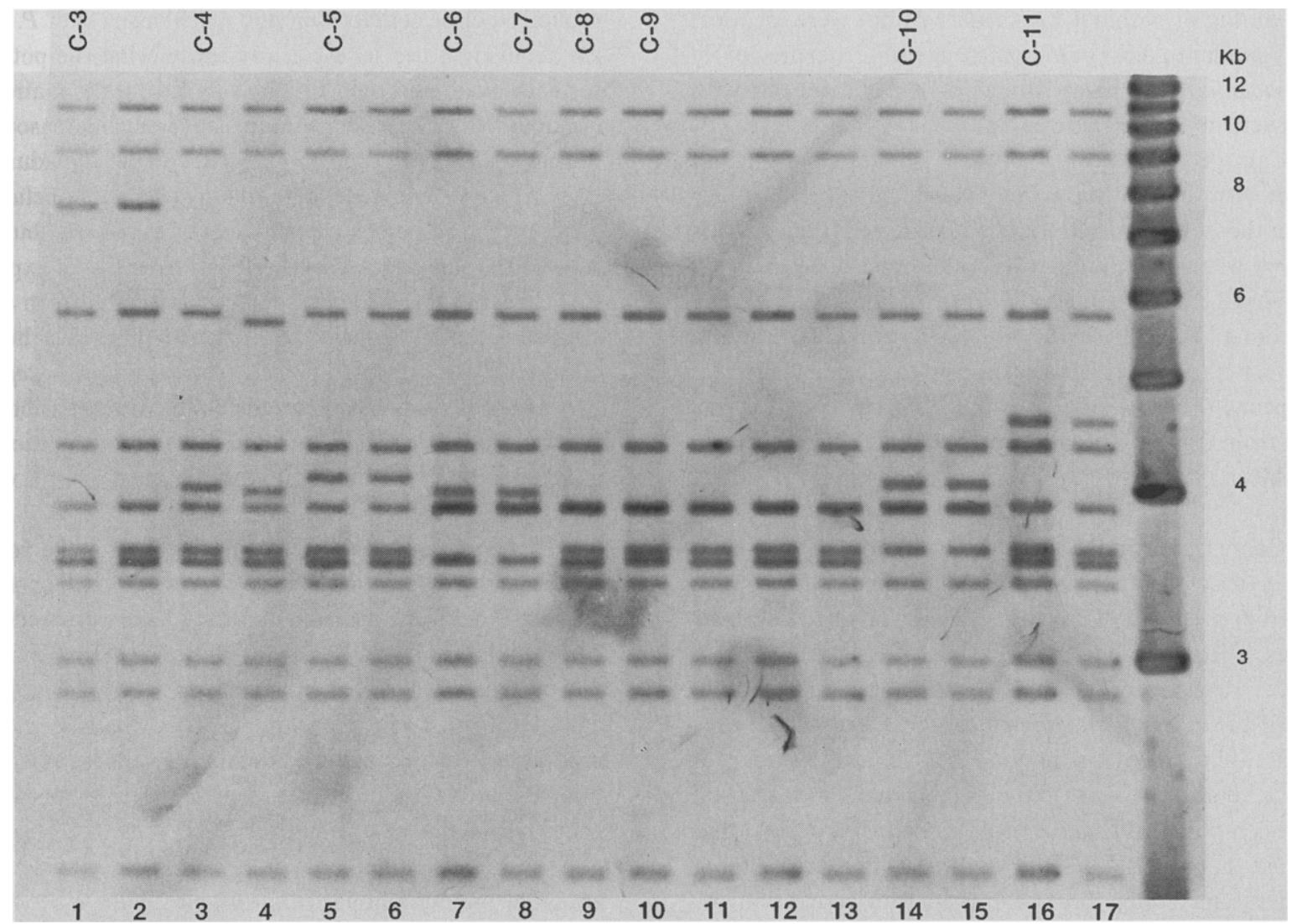

Fig. 2. Ribotyping of Cla I-digested DNA from P. cepacia isolates, Camp C, Ontario. P. cepacia from nine Camp C campers who acquired $P$. cepacia: C3 (lane 1), C4 (lane 3), C5 (lane 5), C6 (lane 7), C7 (lane 8), C8 (lane 9), C9 (lane 10), C1O (lane 14), CII (lane 16); and isolates from other P. cepacia-infected Camp C campers (lanes 2, 4, 6, II to 13, 15, and 17). Size markers in kilobase pairs = migration of HindIII-digested lambda phage DNA (lane 18). The P. cepacia isolates from subjects with conversion $C 3$ (lane 1) and $C 9$ (lane 10) had ribotypes identical to those of isolates from campers (lanes 2 and 11, respectively) with whom they reported close contact for the duration of camp. The ribotype of the $P$. cepacia isolate from converter $C 9$ also matched that of $P$. cepacia isolates from another converter (C8, lane 9) and two other known $P$. cepacia-infected campers (lanes 12 and 13); however, converter $\mathrm{C} 9$ did not report close contact with these individuals at camp, and they attended different CF centers. The seven remaining subjects with sputum conversion had $P$. cepacia isolates with ribotypes that matched those of isolates from $P$. cepacia-infected Camp C attendees (including those with conversion during camp) with whom no close contact was reported: $C 4$ (lane 3) and lane 4, with one band difference among 12 bands; $C 5$ (lane 5) and lane 6; Cl0 (lane 14) and lane 15; Cll (lane 16), and six other campers (common ribotype shown in lane 17); and converters $C 6$ (lane 7) and $C 7$ (lane 8) who had no documented exposure between them and were from different $\mathrm{CF}$ centers. The latter ribotype was not found in any of the available isolates from 33 of $37 P$. cepacia-infected campers.

tributions (male subjects: 95 [52\%] of 181 campers with negative results vs 47 [51\%] of 92 control subjects), and similar proportions of patients whose cultures colonized $P$. aeruginosa organisms in 1988 to 1989 (114 [63\%] of 181 vs $66[72 \%]$ of 92 , respectively; $p=0.15$, chi-square test).

Risk factors for $\boldsymbol{P}$. cepacia acquisition at camp. The 11 subjects with sputum conversion did not differ from the 180 without conversion in age, sex, or severity of underlying CF. Data from camp questionnaires that were completed by 9 of 11 subjects with sputum conversion and 170 of 180 without conversion revealed that the risk of sputum conversion was significantly higher among those who reported having slept in the same cabin, shared an item, or danced with a $P$. cepacia-infected camper (Table). The risk of $P$. cepacia acquisition was not significantly greater among those who reported having hugged or kissed a $P$. cepacia-infected camper or washed hands infrequently, and was significantly less for those who reported having eaten fresh fruit or vegetables (Table).

Ribotyping. Restriction fragment length polymorphisms (i.e., ribotypes of $P$. cepacia isolates from all 11 campers whose sputum culture or cultures became positive for $P$. $c e$ - 
pacia during or within 4 weeks after camp) were identical or very similar to those of $P$. cepacia isolates from previously $P$. cepacia-infected camp attendees or from subjects with co-conversion in the same camp.

In Camp B, one of two P. cepacia isolates from the first subject with sputum conversion had the same banding pattern as the isolate from the second converter (Fig. 1). This ribotype was very similar to (i.e., had one band different from, and 11 bands identical with) the ribotype of an isolate from a known $P$. cepacia-infected camper with whom the two with sputum conversion shared sleeping quarters and spent $>4$ hours a day at camp. This ribotype was different from the common ribotype of $P$. cepacia isolates from the other nine known $P$. cepacia-infected Camp B participants.

At Camp C, all nine subjects with sputum conversion had $P$. cepacia isolates that closely matched the ribotype of isolates from previously known $P$. cepacia-infected camp attendees or from those with co-conversion (Fig. 2). Of these nine, two (converters $\mathrm{C} 3$ and $\mathrm{C} 9$ ) had ribotypes identical to those of the $P$. cepacia-infected campers with whom they shared cabins, spent $>4$ hours a day for the duration of camp, or both. The seven remaining subjects with sputum conversion had $P$. cepacia isolates with ribotypes that matched those of isolates of $P$. cepacia-infected Camp C attendees (including those whose sputum conversion occurred at camp) with whom no close contact was reported.

Water samples. Of the 36 water samples obtained from the three study camps, only a specimen of ice water obtained from a picnic jug at Camp B contained P. cepacia. However, the ribotype of this P. cepacia isolate was different from that of isolates from the two Camp B subjects with sputum conversion (Fig. 1). No P. cepacia organisms were isolated from the other samples, which included water from sinks $(n=12)$, showers $(n=10)$, lakes and ponds $(n=5)$, swimming pools $(n=5)$, and drinking fountains $(n=3)$.

\section{DISCUSSION}

Nosocomial acquisition of $P$. cepacia by patients with CF has been strongly suggested, ${ }^{3,9}$ but $P$. cepacia acquisition at patients' homes, outpatient clinics, and summer camps has not been well studied. ${ }^{6,10,11,15}$ The epidemiologic and laboratory findings of this investigation strongly suggest that $P$. cepacia can be acquired at CF summer camps, and that one of the possible modes of $P$. cepacia transmission at camp is by person-to-person contact.

Previous studies suggest that person-to-person transmission is one likely mode of $P$. cepacia acquisition. ${ }^{2,} 8-11$ In thisinvestigation, we found that campers with sputum conversion were more likely than those with no conversion to have reported specific exposures to other $P$. cepacia-infected campers. These exposures are more likely to be proxies for prolonged close contact than the mechanism(s) of $P$. cepacia acquisition per se, espeically considering the potential for both recall and reporting bias in the questionnaire. We also found that the risk of sputum conversion increased with the prevalence of $P$. cepacia-infected campers and duration of camp. These epidemologic findings do not conclusively prove person-to-person transmission of $P$. cepacia, but they suggest that an increased duration or intensity of exposure to $P$. cepacia-infected campers was associated with an increased risk of $P$. cepacia acquisition at camp and they are consistent with person-to-person transmission of $P$. cepacia.

A previous study has reported possible transmission of $P$. cepacia from $P$. cepacia-infected to uninfected patients at a 7-day camp. ${ }^{15}$ Within 3 months after camp, 9.1\% of patients susceptible to $P$. cepacia infection acquired $P$. cepacia. However, because none of these campers had a positive sputum culture result on the last day of camp, the possibility that $P$. cepacia transmission may have occurred after camp could not be excluded. In addition, the risk of $P$. cepacia acquisition among noncampers was not rigorously assessed. In studies of transmission of $P$. aeruginosa (a microorganism with similar epidemiologic characteristics in patients with $\mathrm{CF}$ ) in $\mathrm{CF}$ summer campers, transmission could not be demonstrated. ${ }^{16,17}$

Nosocomial outbreaks of $P$. cepacia respiratory colonization or infection in patients without $\mathrm{CF}$ have been associated with environmental contamination of disinfectants, antiseptics, and water. ${ }^{1,2,4,18-20}$ However, an environmental source did not appear to be responsible for $P$. cepacia transmission in the study camps. Respiratory therapy devices should not have been involved in $P$. cepacia transmission at the camps, because these devices were not shared between patients and were routinely discarded after less than 24 hours of use by a single person.

In this investigation, we used sputum conversion from negative to positive culture results as the indicator of new $P$. cepacia acquisition. Upper airway culture is a sensitive method of detecting established lower airway, non- $P$. cepacia bacterial colonization in patients with $\mathrm{CF} .{ }^{21,22}$ However, the sensitivity of sputum or throat culture methods in detecting new lower airway acquisition of $P$. cepacia is not known and may be affected by factors such as specimen handling and processing, the density of microorganisms in the lower airway, the severity of the patient's lung disease, and the patient's effort to cough. Anecdotal accounts have suggested that sputum culture methods might not be highly sensitive in prompt identification of new or intermittent $P$. cepacia colonization in patients with $\mathrm{CF}^{8,11}$ although $P$. cepacia transmission from patients with $\mathrm{CF}$ who have negative culture results has not been shown. Application of new techniques, such as use of the polymerase chain reaction, may improve the detection of new $P$. cepa- 
cia acquisition in the respiratory tract and help to elucidate the mechanisms and risks of transmitting $P$. cepacia between $C F$ patients. In the interim, however, in response to the concerns noted above, as well as the results of this and other camp and noncamp studies, the U.S. Cystic Fibrosis Foundation issued a recommendation to discontinue $\mathrm{CF}$ summer camps (U.S. CF Center Directors Committee, 1993).

We used ribotype analysis to link subjects with sputum conversion and the $P$. cepacia-infected campers. However, nonparticipation in the study by some campers limited our ability to assess completely the ribotype patterns at Camp C. Of the 24 Camp C campers who did not consent to participate in the study, 4 were known to have $P$. cepacia infection and could have served as sources of $P$. cepacia. In addition, the use of a weekly rather than a daily exposure questionnaire may have limited the ability of campers attending Camp $\mathrm{C}$ to recall and enumerate contacts with other campers for each 7-day period.

In the acute care hospital setting, the use of contact isolation precautions for the prevention of nosocomial transmission of multi-drug-resistant bacteria, including $P$. cepacia, has been recommended by the CDC. ${ }^{23}$ This study did not assess the degree to which contact isolation precautions were observed at each camp. Thus the impact of these precautions on reducing the risk of $P$. cepacia transmission at camp or in other settings outside the hospital has not been determined. However, strict observance of contact isolation precautions or cohort assignments of campers with $\mathrm{CF}$ by their $P$. cepacia colonization status at CF summer camps or other settings outside the hospital may be impractical and may impair the social and interactive purpose of such gatherings.

On the basis of results of this and other camp studies, the CDC has recommended that in CF camps where transmission of $P$. cepacia has been suspected, or where the expected prevalence of $P$. cepacia-infected campers is $>5 \%$, and/or where the duration of camp is longer than 1 week, the following should be considered: either full implementation of contact isolation precautions at the CF summer camp or prohibition of attendance of $P$. cepacia-infected and uninfected patients at the same CF camp at the same time. In areas with a high prevalence of $P$. cepacia-infected patients, separate camps for infected and uninfected patients may be feasible. ${ }^{24}$

These recommendations depend on adequate procedures for screening patients before camp. Therefore, until more sensitive methods of detecting $P$. cepacia in the respiratory tract of CF patients become available, it is essential that sputum or throat culture specimens be appropriately collected from patients with $\mathrm{CF}$ and transported to and processed in a laboratory that routinely uses $P$. cepacia-se- lective media, and by personnel who are proficient in isolating and identifying $P$. cepacia from sputum of patients with $\mathrm{CF}^{25}$

The recommendations discussed above represent the minimum precautions to be taken if CF summer camps are to be held, because the risk of transmission, albeit small, may be present in camps where an infected patient is in attendance. The recommendations are not meant to restrict CF centers or summer camps from requiring additional precautions or adopting a more stringent approach.

Participants in the Summer Camp Study Group are as follows: William Spohn, MD, Donna Diakew, MS, Children's Medical Center, Dayton, Ohio; Karen McCoy, MD, Terri Johnson, RT, Columbus Children's Hospital, Columbus, Ohio; Robert W. Wilmott, MD, Vikki L. Kociela, RN, Kim Bivens, RN, MSN, Children's Hospital Medical Center, Cincinnati, Ohio; Jamshed F. Kanga, MD, University of Kentucky Medical Center, Lexington, Ky.; John Christenson, MD, Carol Woods, RN, University of Utah Medical Center, Salt Lake City, Utah; Joseph Reisman, MD, Louise Ciccale-Taylor, BScN, Hospital for Sick Children, Toronto, Ontario, Canada; W. M. Wilson, MD, Rosamund Hennessey, RN, Children's Hospital at Chedoke-McMaster, Hamilton, Ontario, Canada, E. R. Eccelstone, MD, Elizabeth Hunter, BScN, Children's Hospital of Western Ontario, London, Ontario, Canada; and Kathryn Keely, MD, Children's Hospital of Eastern Ontario, Ottawa, Ontario, Canada.

We thank Anna Muzinski-Kwan, Douglas Crozier, MD, Margaret Pease, RN, and the staff members of the camps for their help and cooperation during this investigation.

\section{REFERENCES}

1. Tablan OC, Chorba TL, Schidlow DV, et al. Pseudomonas cepacia colonization in patients with cystic fibrosis: risk factors and clinical outcome. J PEDIATR 1985;107:382-7.

2. Tablan OC, Martone WJ, Doershuk DF, et al. Risk factors and outcomes associated with Pseudomonas cepacia colonization of the respiratory tract of patients with cystic fibrosis. Chest 1987;91:527-53.

3. Thomassen MJ, Demko CA, Klinger JD, et al. $\boldsymbol{P}_{\text {seudomonas }}$ cepacia colonization among patients with cystic fibrosis: a new opportunist. Am Rev Respir Dis 1985;131:791-6.

4. Isles A, Maclusky I, Corey M, et al. Pseudomonas cepacia infection in cystic fibrosis, an emerging problem. J PEDIATR 1984;104:206-10.

5. Lewin LO, Byard PJ, Davis PB. Effect of Pseudomonas cepacia colonization on survival and pulmonary function of cystic fibrosis patients. J Clin Epidemiol 1990;43:125-31.

6. Tablan OC and the National Surveillance of Pseudomonas cepacia Study Group. National surveillance for Pseudomonas cepacia [Abstract]. Pediatr Pulmonol 1989;4(suppl):138.

7. FitzSimmons SC. The changing epidemiology of cystic fibrosis. J Pediatr 1992;122:1-9.

8. LiPuma JJ, Dasen SE, Nielson DW, et al. Person-to-person transmission of Pseudomonas cepacia between patients with cystic fibrosis. Lancet 1990;336:1094-6. 
9. Pegues DA, Tablan OC, Schidlow DV, et al. Nosocomial transmission of Pseudomonas cepacia in cystic fibrosis patients [Abstract]. Pediatr Pulmonol 1990;5(suppl):248-9.

10. Thomassen MJ, Demko CA, Doershuk CF, et al. Pseudomonas cepacia: decrease in colonization in patients with cystic fibrosis. Am Rev Respir Dis 1986;134:669-71.

11. Govan JRW, Brown PH, Maddison J, et al. Evidence for transmission of Pseudomonas cepacia by social contact in cystic fibrosis. Lancet 1993;342:15-8.

12. Gilligan $\mathrm{PH}$, Gage PA, Bradshaw LM, et al. Isolation medium for the recovery of Pseudomonas cepacia from respiratory secretions of patients with cystic fibrosis. J Clin Microbiol 1985; 22:5-8.

13. Gilardi GL. Identification of Pseudomonas and related bacteria. In: Gilardi GL, ed. Glucose nonfermenting gram-negative bacteria in clinical microbiology. West Palm Beach, Florida: CRC Press, 1978:15.

14. Standard methods for the examination of water and wastewater. 17th ed. New York: American Public Health Association, 1989.

14a. Hinojosa-Ahumada M, Swaminathan B, Hunter SB, et al. Restriction fragment length polymorphisms in rRNA operons for subtyping Shigella sonnei. J Clin Microbiol 1991; 29:2080-4.

15. Tablan OC, Wilmott RW, Carson LA, et al. Epidemiology of Pseudomonas cepacia at a cystic fibrosis summer camp [Abstract]. Program and abstracts of the 28 th Interscience Conference on Antimicrobial Agents and Chemotherapy. Washington, D.C.: American Society for Microbiology, 1988:359.

16. Speert DP, Lawton D, Damm S. Communicability of Pseu- domonas aeruginosa in a cystic fibrosis summer camp. J PEDIATR 1982;101:227-9.

17. Smith DL, Smith EG, Stableforth DE, et al. Pseudomonas aeruginosa epidemiology: use of two genetic probes in a summer camp study [Abstract]. Pediatr Pulmonol 1991;6(suppl):281.

18. Martone WJ, Tablan OC, Jarvis WR. The epidemiology of nosocomial epidemic Pseudomonas cepacia infections. Eur J Epidemiol 1987;3:222-32.

19. Tablan OC, Martone WJ, Jarvis WR. The epidemiology of Pseudomonas cepacia in patients with cystic fibrosis. Eur J Epidemiol 1987;3:336-42.

20. Hardy KA, McGowan KL, Fisher MC, Schidlow DV. Pseudomonas cepacia in the hospital setting: lack of transmission between cystic fibrosis patients. J PEDIATR 1986;109:51-4.

21. Thomassen MJ, Klinger JC, Badger SJ, et al. Cultures of thoracotomy specimens confirm usefulness of sputum cultures in cystic fibrosis. J PEDIATR 1984;104:352-6.

22. Konstan MW, Hilliard KA. Comparison of throat with bronchoalveolar lavage cultures in determining lower airway bacterial colonization in cystic fibrosis [Abstract]. Pediatr Pulmonol 1991;6(suppl):281.

23. Garner JS, Simmons BP. Guideline for isolation precautions in hospitals. Infect Control 1983;4(suppl):245-325.

24. Centers for Disease Control. Pseudomonas cepacia at summer camps for persons with cystic fibrosis. MMWR 1993;42:456-9.

25. Tablan OC, Carson LA, Cusick LB, Bland LA, Martone WJ. Use of selective media in isolating Pseudomonas cepacia from simulated sputum specimens of cystic fibrosis patients: results of laboratory proficiency tests. J Clin Microbiol 1987;25:485-7. 